\title{
BRAIN MAGNETIC RESONANCE IMAGE LATERAL VENTRICLES DEFORMATION ANALYSIS AND TUMOR PREDICTION
}

\author{
Kai Xiao ${ }^{1}$, Sooi Hock $\mathrm{Ho}^{2}$, Aboul Ella Hassanien ${ }^{3}$ \\ ${ }^{1,2}$ Faculty of Engineering and Computer science, \\ University of Nottingham, Malaysia Campus , 43500 Selangor, Malaysia, \\ Email: kcxk2x@nottingham.edu.my \\ ${ }^{2}$ Faculty of Engineering and Computer science, \\ University of Nottingham, Malaysia Campus, 43500 Selangor, Malaysia, \\ Email: Ho.Sooi-Hock@nottingham.edu.my \\ ${ }^{3}$ Information Technology Department, Faculty of Computer and Information, \\ Cairo University, 5 Ahmed Zewal St., Orman, Giza, Cairo, Egypt. \\ Email: a.hassanien@fci-cu.edu.eg
}

\begin{abstract}
Brain tumor detection is still a challenge in the field of brain compute-aided diagnosis. In the brain Magnetic Resonance Images (MRI), the correlation between lateral ventricles deformations and tumor existence has been found useful in brain tumor detection and prediction. To retrieve the lateral ventricles deformation data for further statistical analysis and processing, a new method has been proposed in this paper to analyze the deformation of ventricles. Firstly, in this method, the boundaries of the lateral ventricles are segmented, pixels on the boundary are sampled, and a nonlinear interpolation method based on Thin Plate Spline (TPS) is conducted to create a more accurate template image for each specific case, followed by the application and performance comparison between TPS with Radial Basis Function Neural Networks (RBF-NN) and Radon Transform (RT) on the extracted Skeleton of the boundary of the ventricles for locating the optimal orientation of the image through iterative image rotation. The reorienting facilitates the final step of deformation analysis whereby the reoriented ventricles are analyzed based on the displacement values obtained from the TPS of the sampled template and the diagnostic lateral ventricle. By comparing with several real cases, our experimental results suggest that this method is effective and relevant in ventricles deformation analysis and prediction of tumor location. The performance comparison results also suggest that using RT on Skeleton is an efficient method in locating the optimal orientation where the results show that the computing speed is at least more than 100 times faster than using TPS and RBF-NN.
\end{abstract}

Keywords: Magnetic Resonance Imaging, Lateral Ventricles, Deformation Analysis, Thin Plate Spline, Morphing, Radon Transform, Skeleton, Radial Basis Function

\subsection{INTRODUCTION AND MOTIVATION}

The objective of this work is to construct a scheme for predicting the approximate location of brain tumors based on the deformation of the lateral ventricle boundary, without studying any property of brain tumor, to provide assistance for further work on brain compute-aided diagnosis of brain tumor and segmentation.

It is found that there is a correlation between shape deformation of the brain ventricular compartments and the probability of tumor existence. As a case study, if there is a blockage of the ventricular system causing the flow of CSF interrupted, for example, a blockage within the cerebral aqueduct, the normal flow of fluid formed in the lateral ventricles and the third ventricle is interrupted, and the lateral ventricles and third ventricle begin to swell with CSF. The swelling or enlargement is termed hydrocephalus. Hydrocephalus can also result from the formation of CSF (as can occur with a tumor in one of the choroid plexuses) that exceeds the amount that can flow through the ventricular system, or from a downstream-diminished capacity to absorb CSF [1]; therefore, studying the lateral ventricles deformation becomes a useful assistance to certain cerebral diseases diagnosis.

As can be discerned from Fig. 1, when one or more tumors exist, though being irregular with respect to the physical and chemical properties of the existing brain tumors, the shape of the lateral ventricles will be deformed by the compression from the growth of brain tumor. From the observations in some cases, part of the ventricle shrinks to the opposite direction of the brain tumor. 
Therefore, finding out the deformation of the lateral ventricular compartment is useful in making an estimation or prediction to the probability and location of the existence of brain tumor. As can be seen from the healthy brain MRI in Fig. 1 (f), the shapes of the left and right lateral ventricular compartments are similar to each other. However, in the cases where brain tumor exists as in Fig. 1 (a - e), the compression from tumor which leads to lateral ventricle deformation will normally affect more on one ventricle, and create an imbalance on the shape of the two ventricles. Hence, the shape difference between the left and right lateral ventricles is offering an additional indicator in estimating the existence of brain tumor.

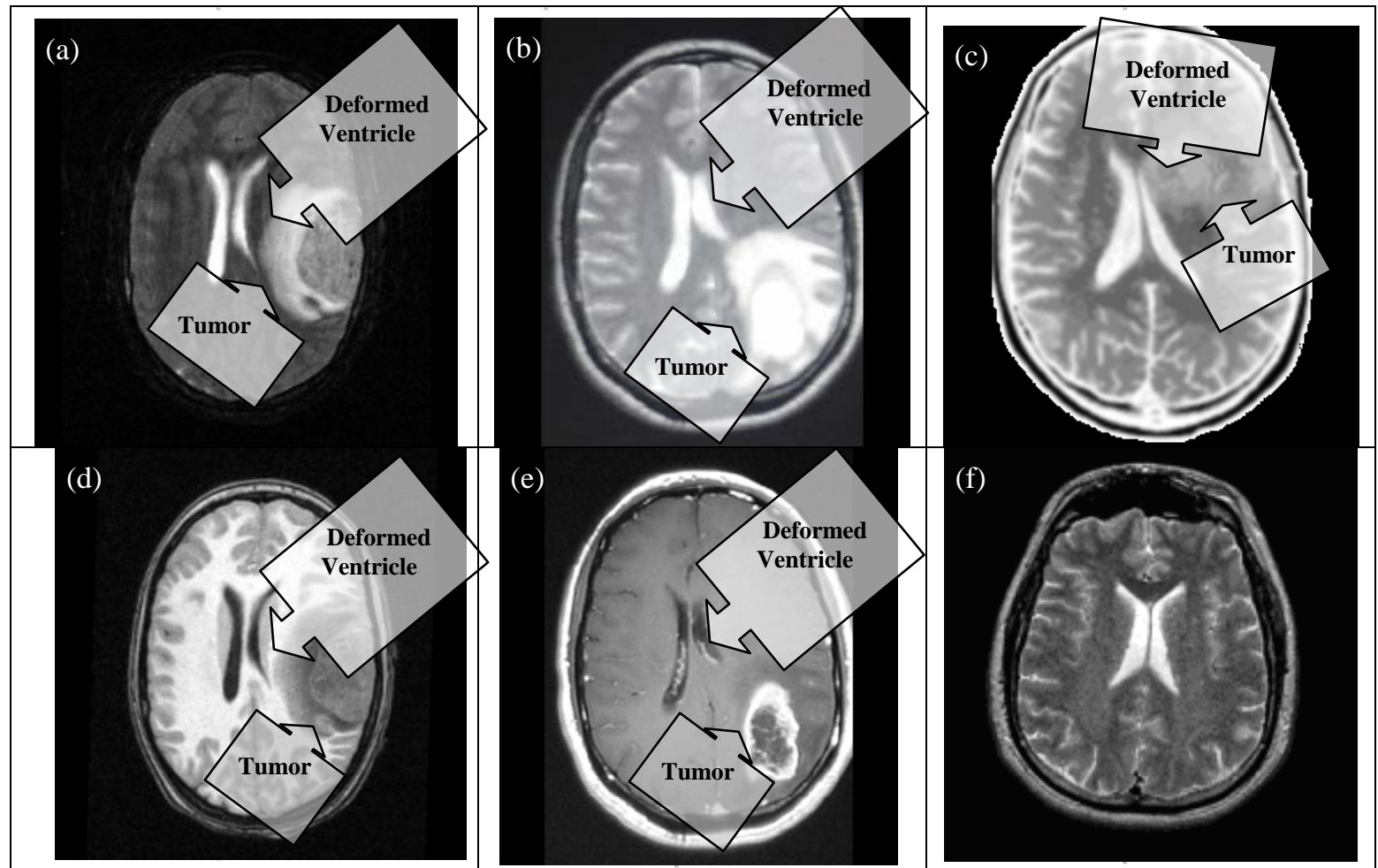

Fig. 1:(a,d) T1 and T2 weighted MR images with tumor [2], (b,e,c) T1 and T2 weighted MR images with tumor[3], (f)T1 weighted MR image of normal brain [4]

From the problems related with brain MR images segmentation that was discussed in our previous work [5][6], factors such as noise, spurious blobs, inhomogeneous pixel intensity distribution, and blunt region boundary which contribute to the complexity in the segmentation are distributed in the whole brain MR images. This creates greater challenges to brain tumor detection and segmentation. Even by some advanced methods, those problems are only able to be alleviated, but are not able to be fully solved due to the nature of MR images retrieval. Therefore, minimizing the brain tumor detection scope will be a desirable approach in solving the problems.

It is then reasonable to take advantage of the correlations between ventricles shape deformation and the existence of brain tumor to shrink the region down to reduce the tumor detection and segmentation workload. In order to get the optimized ventricle deformation analysis results, desirable methods should be selected or designed.

In the pioneering works related with image deformation analysis, Gaser et al. [7] used Thin Plate Spline (TPS) for deformation based morphometry on the MRI slices to match the image under diagnosis to the template; For imageguided surgery systems during neurosurgery, Ferrant et al. [8] used a deformable surface matching algorithm to capture the deformation of boundaries of key structures (cortical surface, ventricles and tumor) throughout the neurosurgical procedure, and a linear finite element elastic model to infer a volumetric deformation. To perform analysis on the effect of alcoholism on brain structure, Rohlfing et al. [9] performed two types of morphometry and justified by the invariance of relevant statistics under changes of the spatial and temporal reference coordinate system on the acquired two structural MR brain images; Mardia et al. [10] addressed the problem of the distortion effect produced by different types of non-linear deformation strategies on textured images. The extensive analysis on a deterministic deformation and a landmark based deformation including TPS was also performed in their study; Ross et al. [11] applied TPS and developed an average deformation model in the area of fingerprint matching; Lin and Lin [12] developed a three-dimensional (3D) image deformation technique to integrate multimodality medical 
images by combining the 3D optical flow (OF) estimation and applied TPS transformation on landmarks tracing and geometric deformation.

In this paper, the use of TPS to find the area with the most significant displacement is proposed. The lateral ventricle boundary is obtained after the lateral ventricular compartments are segmented by the modified Fuzzy-C means algorithm in our previous work [5][6]. Due to the discrepancy in age, sex, personal brain structure, and even MR image slice number, though similar to each other, shapes of diagnostic and template images may be varied greatly to each. Therefore, an interpolated image should be served as a new template which morphs between the template and diagnostic images to enhance the accuracy for the later processes. A nonlinear morphing method based on TPS [13] is used here to create a more accurate interpolation result.

It is found that the orientation between diagnostic and template MR images is the key for creating an accurate result for the TPS displacement analysis. Therefore, it is important to adjust the diagnostic MR image orientation according to the correlation between the diagnostic and template images before the TPS displacement analysis. Because the TPS output data is based on the displacement between the diagnostic and template ventricles, TPS displacement values can also serve as an error measure to be used in the process of finding the best orientation match between the template and the diagnostic image. The displacement information from the TPS analysis on the Skeleton [14] has therefore used in our first reorientation method by the Radial Basis Function Neural Network (RBF-NN) as its error measure input.

An alternative method of reorienting is also proposed in this paper which uses Radon Transform (RT). The RT is applied on the Skeleton of the lateral ventricle and the orientation of the diagnostic ventricle Skeleton with the highest RT value will then be matched with the orientation of the template Skeleton with the highest RT value. The two methods of reorienting has been implemented and experimented; its result shall be compared and discussed in this paper. In Section 2 of this paper, the background of the TPS, RBF-NN, and RT methods used in our study are presented. The general overview of the algorithm that has been implemented and experimented is also discussed. The result from the experiments is then presented and discussed in Section 3.

\subsection{EXPERIMENTAL METHODS}

\subsection{Image Interpolation using TPS}

Interpolated images are certain frames retrieved from a sequence of morphed images. Image morphing is an animated transformation of one image to another. It is used in the experiments to reduce negative effect from the shape difference between template and diagnostic images due to discrepancy between age, sex, personal brain structure, and even MR image slice number.

The simplest approach in morphing just linearly fades from one image to the other, i.e. it uses linear interpolation of the gray or color value for the two images at every pixel location. The result is not very impressive as the shape of image objects is in no way accounted for. A better solution is to transform each image so that pixels that belong to the same object-parts are aligned in a common coordinate system. In the experiments, Thin Plate Spline (TPS) is used.

The term TPS originally refers to the physical analogy of bending thin metal plate introduced to geometric design by Duchon [15]. A flat metal plate can be represented by 2-dimensional coordinate $\mathrm{x}$ and $\mathrm{y}$. A bended metal plate will have a displacement perpendicular to the $\mathrm{x}$ and $\mathrm{y}$ coordinate and therefore be represented with 3-dimensional space, $\mathrm{x}, \mathrm{y}$ and $\mathrm{z}$.

The biharmonic equation as shown in Equation 1 has a fundamental solution as shown in Equation 2 [13],

$$
\begin{aligned}
& \Delta^{2} U=\left(\frac{\partial^{2}}{\partial x^{2}}+\frac{\partial^{2}}{\partial y^{2}}\right)^{2} U=0 \\
& z(x, y)=-U(r)=r(x, y)^{2} \ln r(x, y)
\end{aligned}
$$

where $z(x, y)$ refers to the displacement orthogonal to the plane $x-y$. 
TPS is a process of finding a function, $z(x, y)$ which minimizes the bending energy $E$ as in Equation 3 below [13]:

$$
E(z(x, y))=\iint\left[\left(\frac{\partial^{2} z(x, y)}{\partial x^{2}}\right)^{2}+2\left(\frac{\partial^{2} z(x, y)}{\partial x y}\right)^{2}+\left(\frac{\partial^{2} z(x, y)}{\partial y^{2}}\right)^{2}\right] d x d y
$$

In the case of 2-dimensional coordinate transformation using TPS, only 2-dimensional space is used in which the displacement in the $\mathrm{z}$ direction is reinterpreted as a displacement in the $\mathrm{x}$ and $\mathrm{y}$ coordinate. Fig. 2 below shows the original coordinate of the control points as indicated by the black dot and the surrounding TPS data points arranged as a mesh.

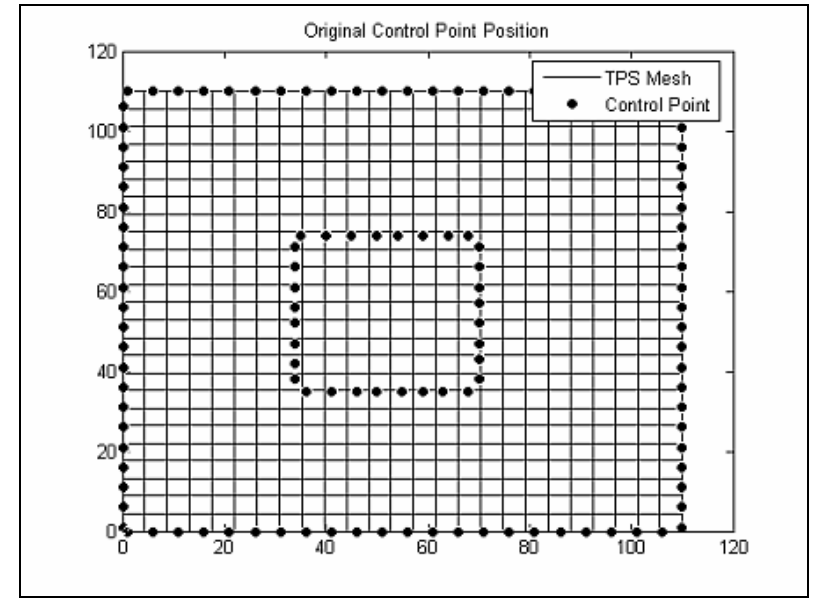

Fig. 2: Initial TPS mesh

The displacement in the $\mathrm{z}$ direction is remapped as a displacement of the original data point coordinate $(\mathrm{x}, \mathrm{y})$ in either $\mathrm{x}$ direction or $\mathrm{y}$ direction or both as shown below:

$$
\begin{gathered}
(x, y) \rightarrow(x, z(x, y)) \\
\text { or } \\
(x, y) \rightarrow(z(x, y), y) \\
\text { or } \\
(x, y) \rightarrow(z(x, y), z(x, y))
\end{gathered}
$$

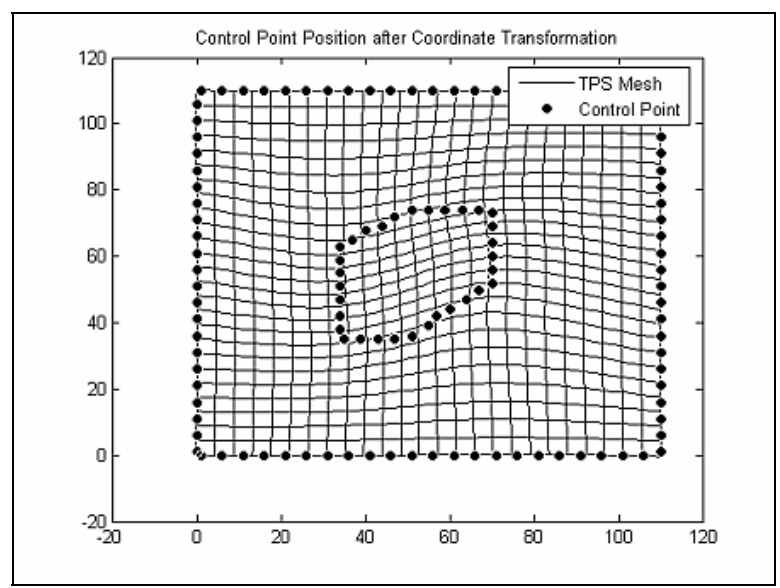

Fig. 3: Deformed TPS mesh 
Fig. 3 above shows that the data points that is originally arranged in a grid pattern is remapped when the control points as indicated by the black dot undergoes coordinate transformation.

In the applications of TPS morphing, TPS is mainly served as a coordinate transformation method. In the experiments, firstly, the corresponding landmark points on the imaged object in the two images are identified. TPS transformation is then estimated from the first to the second for a time parameter, $t \in[0,1]$ with a time step of 0.05 in which the first image is the template and the second image is the diagnostic.

Table 1: Interpolated ventricle boundaries

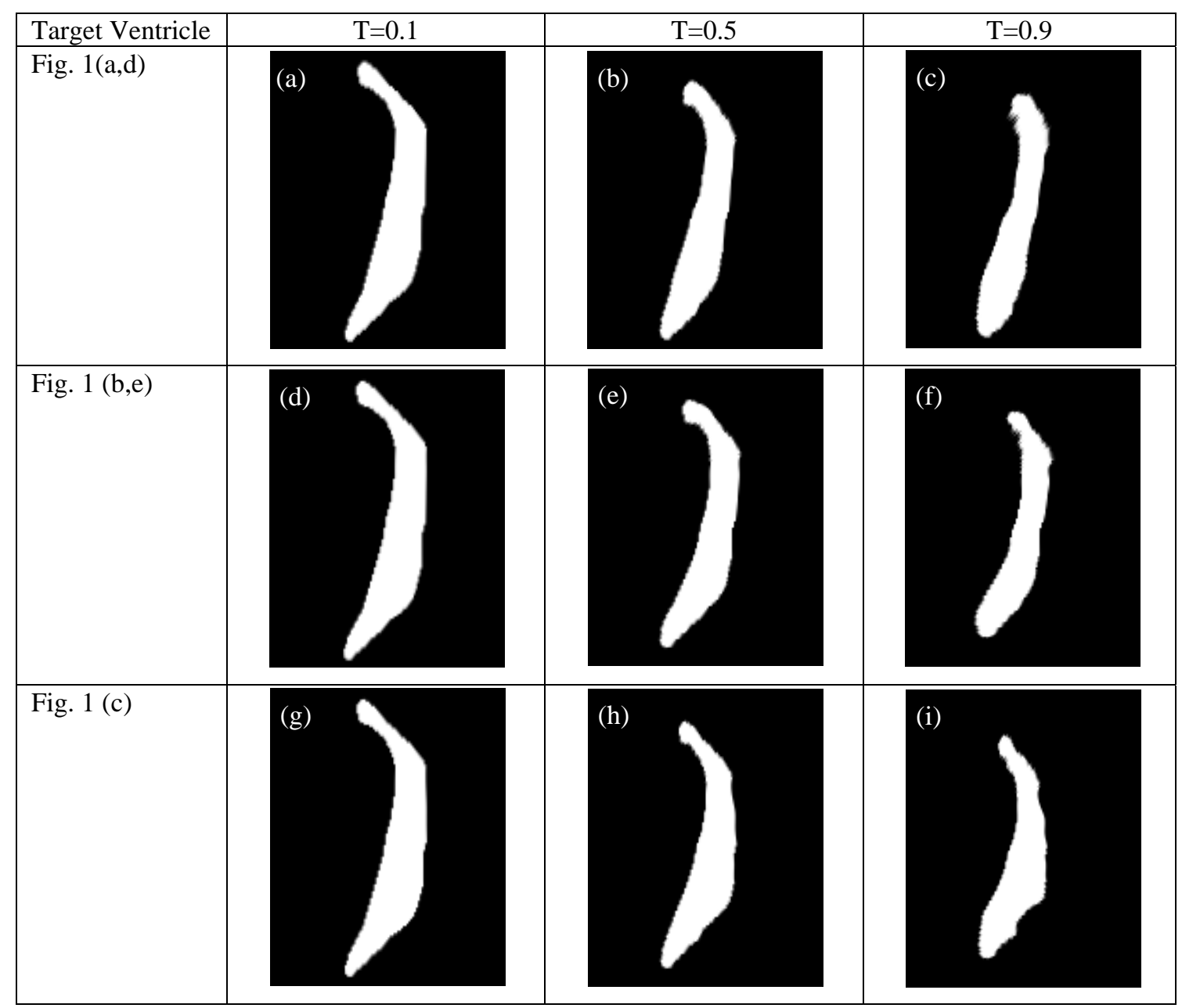

Any image between $t=0$ to $t=1$ can be used as interpolation interval. Interpolated image of time $t=0.5$ is applied in this study as it is the middle image which shares the properties between both images. Table 1 below show the interpolated images between template images as in Fig. 1 (f) to diagnostic ventricles at certain intervals.

After the image interpolation process, template images are replaced by the interpolated images hereafter within this study.

\subsection{Brain Lateral Ventricle Reorientation}

\subsubsection{Reorientation using TPS and RBF-NN}

In the experiments, Radial Basis Function Neural Network (RBF-NN) is applied to the TPS analysis output data which is the TPS displacement value that will be discussed later in this paper. RBF-NN is a type of supervised feed forward neural network that uses Radial Basis Function (RBF) as the activation function in the neural network hidden layer. Fig. 4 below shows the topology of a simple feedforward neural network which consists of the input layer, hidden layer, and the output layer. 


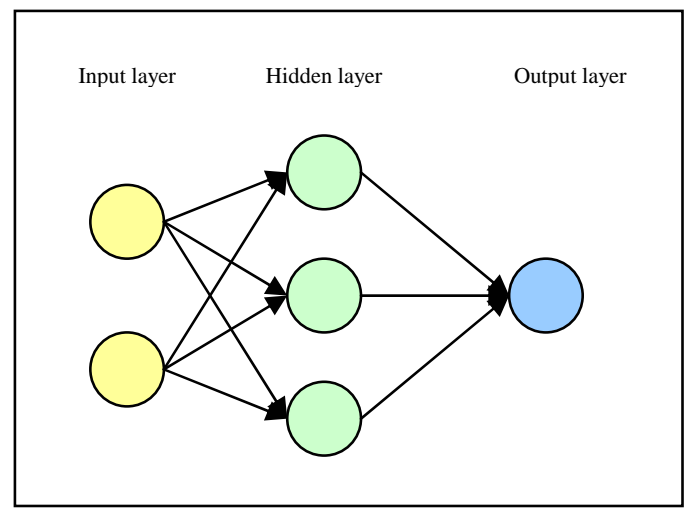

Fig.4: Topology of a simple feedforward neural network

The hidden layer of a RBF-NN consists of nonlinear activation function which is the radial basis function while the output layer consists of linear activation function in which the output is the sum of its weighted inputs.

RBF is a function that operates on any norm which is usually an Euclidean distance of a point, $x$ from a center, c as shown below in Equation 7 [16].

$$
\phi(x, c)=\phi(\|x-c\|)
$$

It can be proven that any function can be approximated using a sum of $\mathrm{N}$ Radial basis functions each with different centers, $C_{i}$ and weights, $w_{i}$ as shown in Equation 8 [17][18].

$$
y(x)=\sum_{i=1}^{N} w_{i} \phi\left(\left\|x-c_{i}\right\|\right)
$$

Equation 8 is a representative of the function of the Radial Basis Function Neural Network. The radial basis function of type Gaussian is used in our reorienting experiment as shown in Equation 9 while the form as shown in Equation 10 is used in deformation analysis experiment using TPS [19],

$$
\begin{aligned}
& \phi(r)=\exp \left(-\beta r^{2}\right) \quad \text { for } \beta>0 \\
& \phi(r)=r^{2} \log (r)
\end{aligned}
$$

where $r$ is the Euclidean distance function.

In the case of $n b$ number of middle layer neuron and 1 output node as used in our experiments, the RBF-NN operation can be summarized as shown in Equation 11 below [19]:

$$
y(x)=\left(\sum_{i=1}^{n b} w_{i} e^{-\lambda_{i}^{2}\left\|x-c_{i}\right\|^{2}}\right)+b
$$

where $y$ is the output of the neural network, $x$ is the input vector to the network, $\lambda$ is the RBF width, $c$ is the center of the RBF, and $w$ and $b$ is the linear layer input weight and bias respectively.

The RBF neural network uses supervised learning where the number of centers, $n b$ and its value, $c$, the input weight to the linear layer, $w_{i}$, and the linear layer bias, $b$, are modified based on the error between the real output, $y$, and the predetermined target output value with a predetermined input vector value, $x$. The learning will stop when the error converges to a low level. 
In the experiments, the RBF-NN is trained using two input vector and two target value. For the first input vector, a vector in which all its elements are zero and for the second input vector in which all its elements is the maximum value of the elements in the expected input vector are used.

The corresponding target output is a value of 0 and a value which is 4 times the maximum value of the elements in the expected input vector. The RBF function width, $\lambda$, is set to 50 times the maximum value of the elements in the expected input vector. The idea is to train the RBF neural network to find a sort of mean of the input vector.

After the RBF neural network is trained, it is then ready to be simulated. By inputting a form of error measure as the input vector, the network can be used for the determination of the input vector with the least error based on its output value.

The Skeleton of the template and diagnostic lateral ventricle which is obtained through morphological operation [14] is used in the TPS analysis to find the displacement between them. Based on the displacement value as its input, the network will produce an output value that will be used for the determination of the orientation with the least displacement.

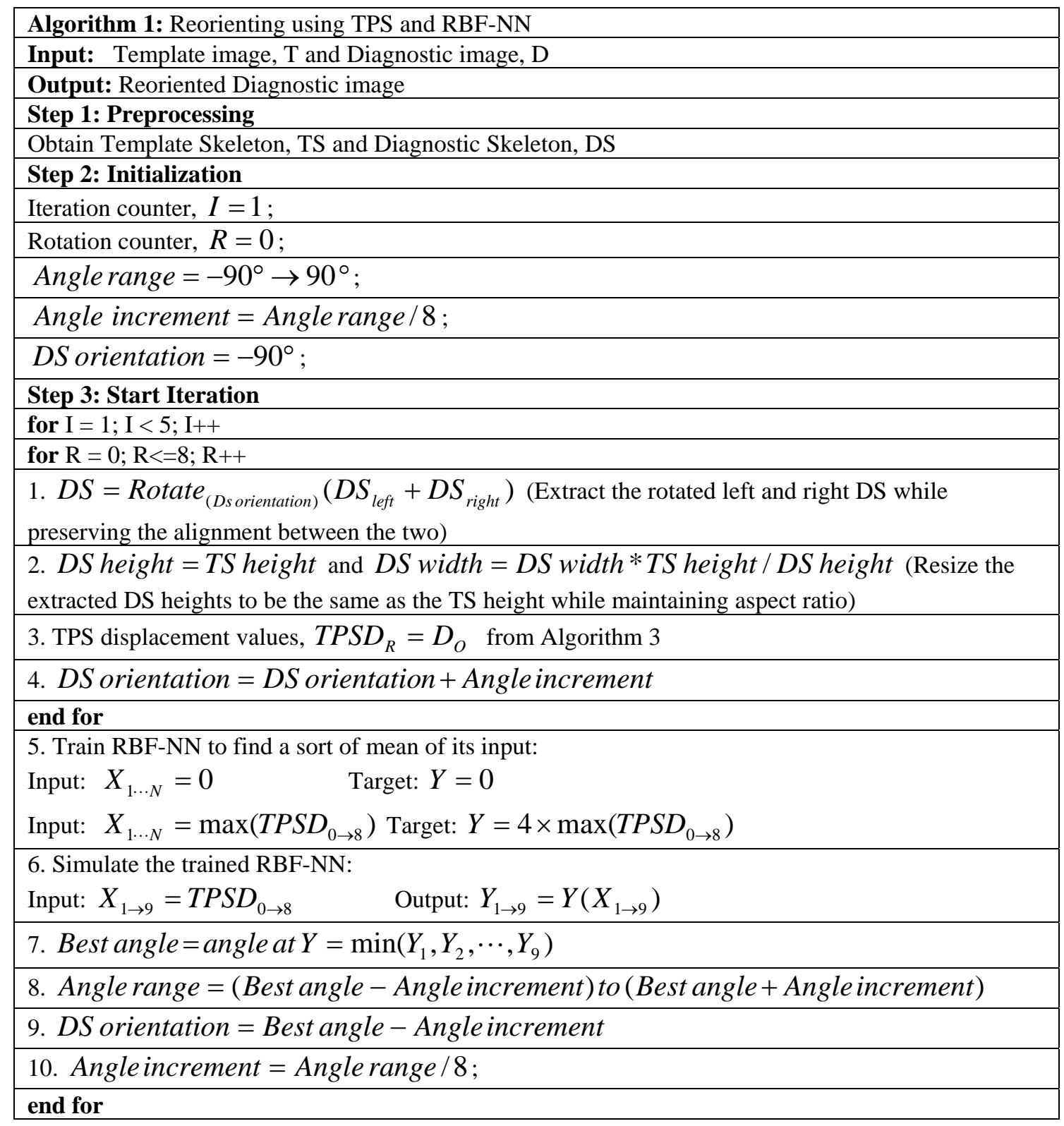


In the experiment, the TPS displacement value is used as an error measure. The initial rotation range is set to -90 to 90 degree. For each iteration, the TPS displacement value between the template and diagnostic Skeleton is calculated for 9 equally spaced angles from the rotation range. The best angle with the least TPS displacement value as found from the output of the RBF-NN will be selected as the center angle of the next reduced rotation range. The iteration proceeds until the $5^{\text {th }}$ iteration and the best angle found will be used to reorient the Diagnostic lateral ventricle. The method used to reorient the diagnostic lateral ventricle image using TPS displacement values as an error measure input for the RBF-NN is shown in Algorithm 1.

\subsubsection{Reorientation using Radon Transform}

Radon Transform (RT) is applied as the second method of reorienting in the experiments. It is based on the principle of skew detection and correction which is similar to the work done by Rajiv et al. [20]. The method uses a common property between the Skeleton of the template and diagnostic which is their longest straight line and tries to match the two together by rotating the Diagnostic Skeleton based on the result of the RT.

$\mathrm{RT}$ is a method of representing a 2D image in $1 \mathrm{D}$ space by the use of straight line projection [21] of the image intensity as illustrated in Fig. 5 below. For each rotation of the image, a line integral of the image in the direction of projection will be performed and the values obtained from each line integral will be the sum of the intensity of the image along the line integral.

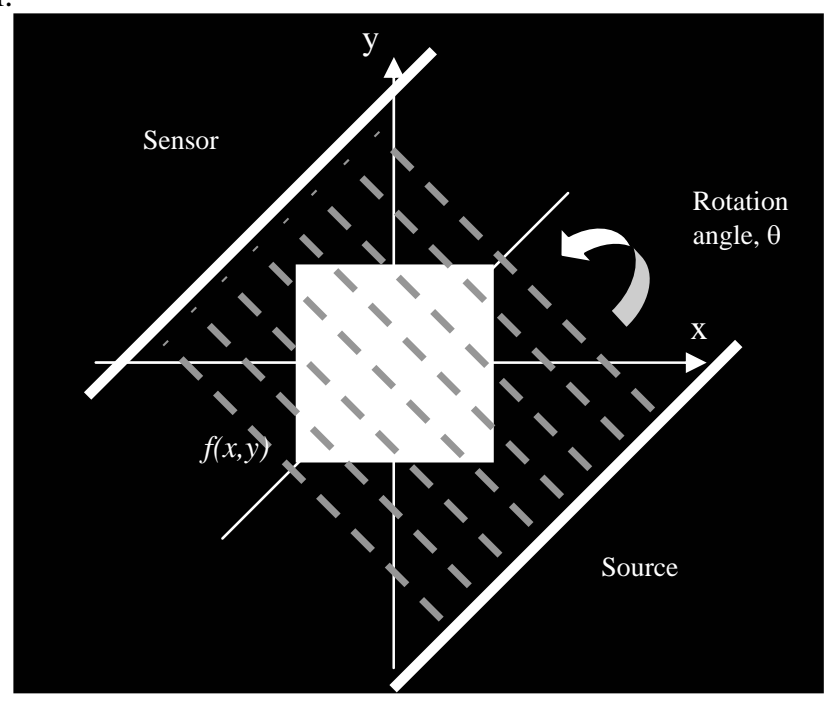

Fig.5: RT - Image Rotation and projection

As seen from Fig. 6 below, the RT will perform line integrals parallel to axis y' on function $f(x, y)$ which represents the intensity of the square image based on its coordinate $\mathrm{x}$ and y to make up the function $R_{\theta}\left(x^{\prime}\right)$. In essence, for 2-dimensional case as shown below, the RT will sum up the intensity that makes up the object matrix along a certain direction depending on the rotation angle, $\theta$ of the $x^{\prime}$ axis.

The Equation 12 below summarizes the general idea of the RT [21],

$$
R_{\theta}\left(x^{\prime}\right)=\iint f(x, y) \delta\left(x^{\prime}-x \cos \theta-y \sin \theta\right) d x d y
$$

where $\delta$ is the Dirac function. 


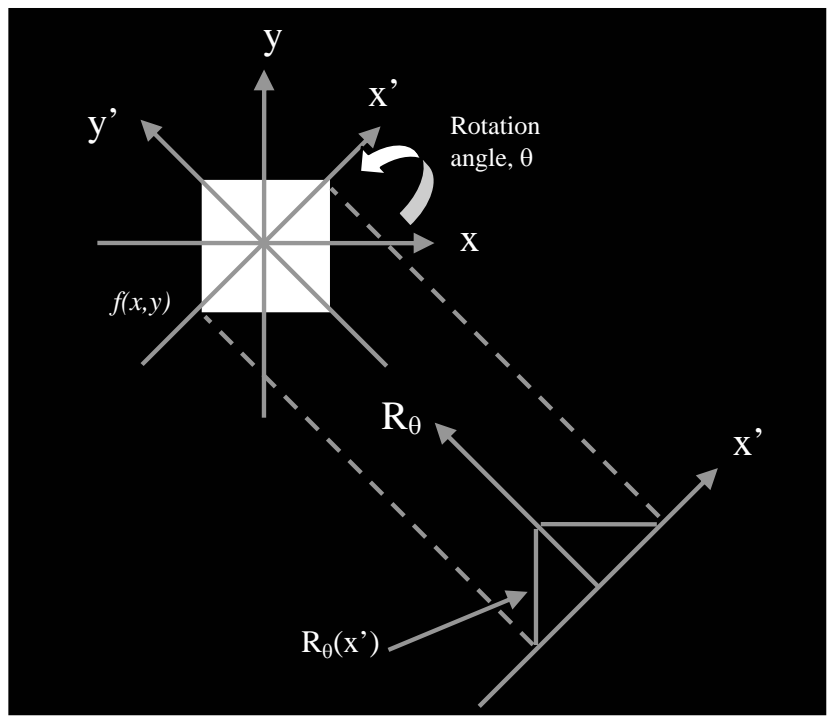

Fig. 6: RT - Line Integral of a square object along y

The method used to re-orientate the diagnostic lateral ventricle image using RT is as shown in Algorithm 2.

\begin{tabular}{|c|}
\hline $\begin{array}{l}\text { Algorithm 2: Reorienting using Radon Transform } \\
\text { Innut: Template image Tand Diagnostic image D }\end{array}$ \\
\hline Output: Reoriented Diagnostic image \\
\hline Step 1: Preprocessing \\
\hline Obtain Template Skeleton, TS and Diagnostic Skeleton, DS \\
\hline Step 2: Initialization \\
\hline $\begin{array}{l}\text { Staring angle }=45^{\circ} ; \\
i=1 ;\end{array}$ \\
\hline Step 3: Start iteration \\
\hline do \\
\hline 1. $D S=$ Rotate $_{(\text {Starting angle) }}(D S)$ (Rotate DS to Starting angle) \\
\hline 2. $R_{\theta=-90 \rightarrow 90}(T S), R_{\theta=-90 \rightarrow 90}(D S)$ (Perform RT on TS and DS) \\
\hline $\begin{array}{l}\text { 3. best angle } \\
\text { best angle } \\
\text { TS }=\text { angle at } \max \left(R_{\theta=-90 \rightarrow 90}(D S)\right) \text {, } \max \left(R_{\theta=-90 \rightarrow 90}(T S)\right) \\
\text { (Find the angle of maximum RT value for TS and DS as template angle and diagnostic angle } \\
\text { respectively) }\end{array}$ \\
\hline 4. Starting angle $=$ Starting angle $+\left(\right.$ best angle ${ }_{T S}-$ best angle $\left.{ }_{D S}\right)$ \\
\hline 5. $i=i+1$ \\
\hline while $\mid$ best angle $e_{T S}-$ best angle ${ }_{D S} \mid \geq 3$ \\
\hline
\end{tabular}

The RT is applied to find the transform at -90 to 90 degrees rotation angle. RT is firstly applied on the left and right Skeleton of the template separately. Following that, the transform of the left and right of the diagnostic Skeleton is then obtained. By analyzing the RT of the template and diagnostic left and right Skeleton, the maximum value of the transform is used for orientation matching purposes. The diagnostic Skeleton is rotated based on the difference between the angle of maximum RT value of the template and the diagnostic. The process of applying the RT and reorienting the Skeleton is performed until the difference in angle of maximum RT value between the template Skeleton and the diagnostic Skeleton is less than three degrees. 


\subsection{Brain Lateral Ventricle Deformation Analysis}

\subsubsection{Deformation Analysis using TPS}

Continued from the discussion on TPS in the previous section, TPS is again served as a tool for deformation analysis. In this experiment, Equation 12 is used to map the displacement from the original data points to the final data points. The original coordinate of the control points are the sampled boundary of the Template, $P_{\text {Template }}$ and the final coordinate of the control points are the corresponding sampled boundary points of the Diagnostic, $P_{\text {Diagnostic }}$ A total of 64 control points are sampled for each left and right ventricle boundary and its Skeleton. The sampling starts at the same vertical position between the template and the diagnostic. The sampling then proceeds in a clockwise direction for the left lateral ventricle and anticlockwise for the right lateral ventricle. By using the standard Euclidean distance function on the original data points and the corresponding final data points, the displacement, $D_{O}$ is shown below in Equation 13:

$$
D_{O}=\sqrt{\left(P_{\text {Original }_{-} x}-P_{\text {Displaced }_{-} x}\right)^{2}+\left(P_{\text {Original }_{-} y}-P_{\text {Displaced }_{-} y}\right)^{2}}
$$

where $P_{\text {Original }}$ is the original data point coordinate, $P_{\text {Displaced }}$ is the final data point coordinate.

Based on the displacement in the $\mathrm{x}-\mathrm{y}$ plane from the original data points, the displacement value is separated into certain ranges. In the current experiment, an active threshold method where the threshold value is between 0 and 1 is used to be multiplied with the maximum displacement value as shown in Equation 14 below:

$$
D_{\text {Range }}=D_{\max } \times \text { threshold }
$$

The original data point that has a displacement value which is within the threshold range, $D_{\text {Range }}$ is plotted with a certain intensity value. In the experiment, 10 different threshold values are used to separate the displacement value to 10 linear ranges between the zero displacement value and the maximum displacement value. The maximum displacement value is marked with a cross in a circle. The area surrounding the mark is postulated to be the source of deformation.

The steps used for deformation analysis using TPS are shown in Algorithm 3.

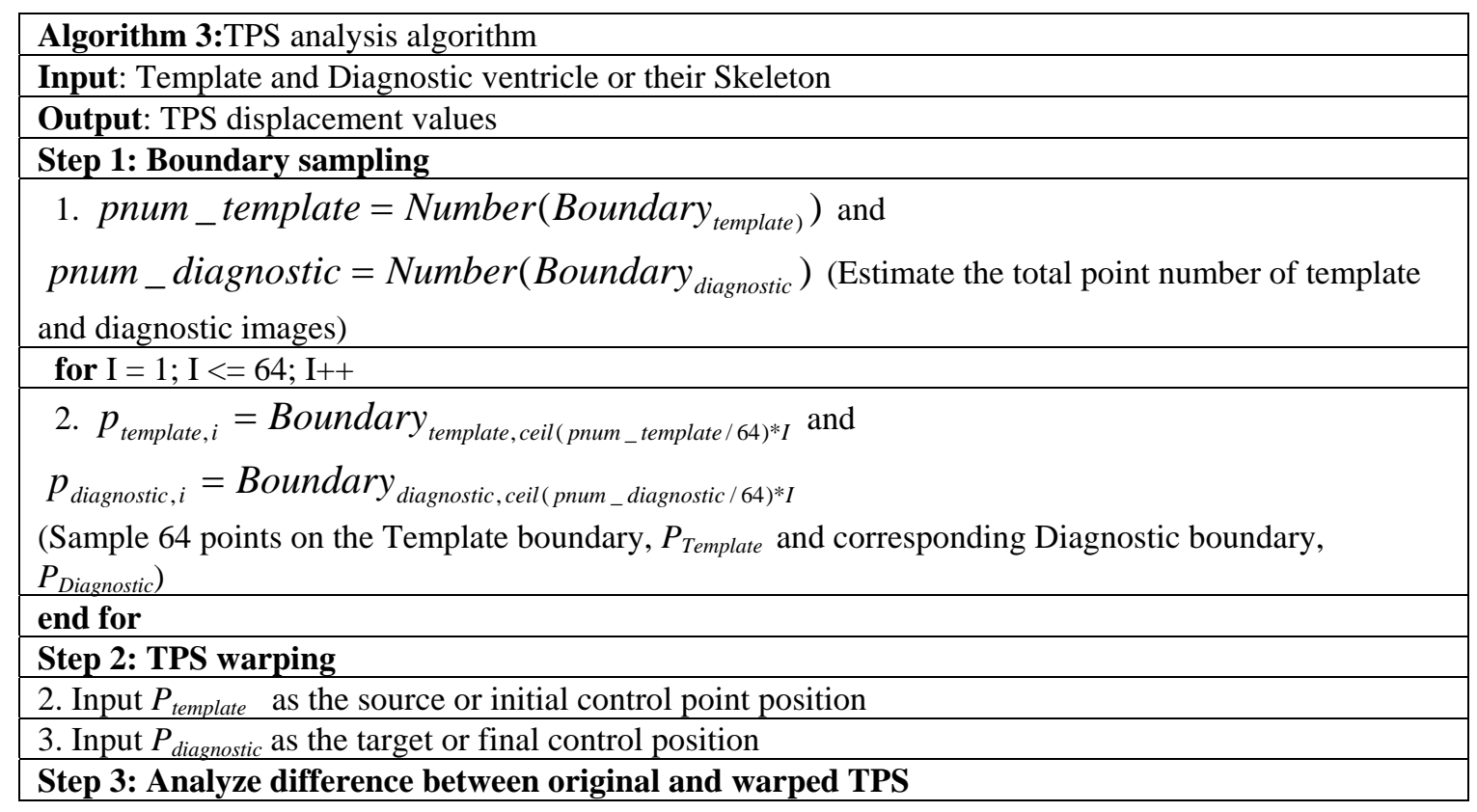




4. From, $E(z(x, y))=\iint\left[\left(\frac{\partial^{2} z(x, y)}{\partial x^{2}}\right)^{2}+2\left(\frac{\partial^{2} z(x, y)}{\partial x y}\right)^{2}+\left(\frac{\partial^{2} z(x, y)}{\partial y^{2}}\right)^{2}\right] d x d y$
and
$(x, y) \rightarrow(z(x, y), z(x, y))$
TPS displacement values, $D_{O}=\left\|P_{\text {Original }}\left(z\left(P_{\text {template }}\right), z\left(P_{\text {template }}\right)\right)-\right\|$
$P_{\text {Displaced }}\left(z\left(P_{\text {diagnostic }}\right), z\left(P_{\text {diagnostic }}\right)\right) \|$
(Find the displacement between the original TPS data points, $P_{\text {Original }}$ and the corresponding displaced
TPS data points, $P_{\text {Displaced }}$

\subsection{RESULTS AND DISCUSSION}

The result shown in Table 2, Table 3, and Table 4 were obtained after reorienting the original diagnostic lateral ventricle image and applying TPS analysis on the re-orientated image. The Skeleton of the template and diagnostic ventricle boundary were used in both the TPS and RBF-NN based reorientation algorithm and RT based reorientation algorithm. In this section, the two reorienting methods are compared and the performance of each is evaluated. The subjective evaluation of the accuracy of the TPS analysis for tumor location predicting is also conducted.

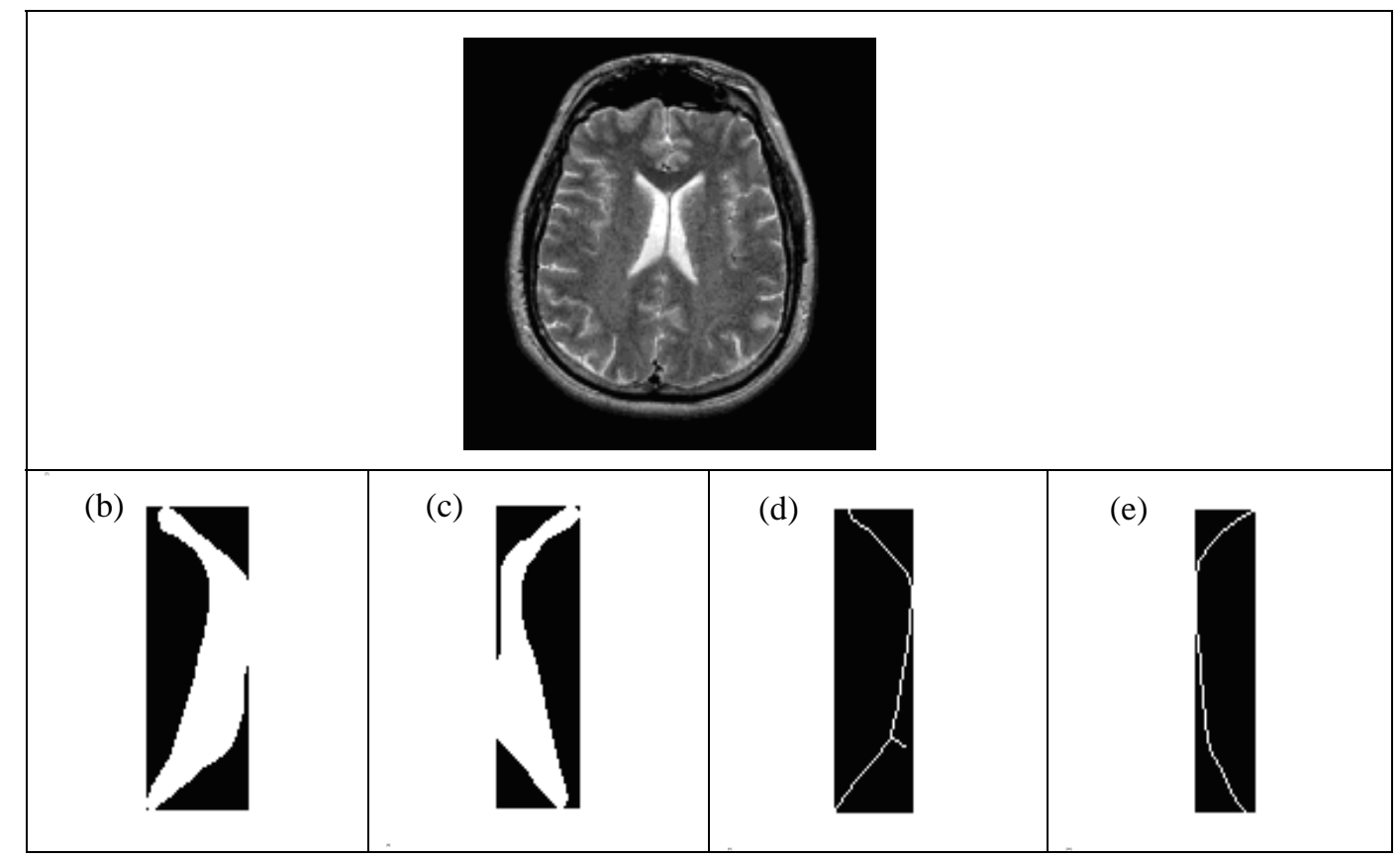

Fig.7: The Template image and its segmented lateral ventricles and ventricle Skeletons

Fig. 7 (a) shows the original template image in which the left and right lateral ventricle in Fig. 7(b) and (c) were extracted from. A morphological operation is then performed on the extracted left and right lateral ventricle to obtain their Skeleton as shown in Fig. 7(d) and (e). The template Skeleton will be the basis in which the orientation of the diagnostic lateral ventricle Skeleton will be compared and adjusted to match with it. 
The result shown in index (a) to (h) in each Table 1 to 3 is obtained after the orientation of the diagnostic lateral ventricle is adjusted to match the template. The degree in which the left and right diagnostic lateral ventricle and its Skeleton is rotated from its original orientation is indicated as adjusted orientation angle in each table.

Based on the adjusted diagnostic lateral ventricle and its Skeleton, TPS analysis is then performed on the sampled boundary point of the lateral ventricle and the Skeleton. From the analysis, the result is obtained as shown in index (i) to (l) which is based on the sampled template and diagnostic boundary points and index (m) to (p) which is based on the sampled template and diagnostic Skeleton boundary points. The area with the highest TPS displacement value is indicated by a cross in a circle mark which indicates the deformation climax predicted from the analysis.

From the result of the TPS analysis on the sampled lateral ventricle boundary using the two reorienting methods, the location of the highest TPS displacement value is determined and its place in the context of its original image is indicated with the same mark as shown in index (q) and (r) respectively.

The processing time of the whole reorienting process and TPS analysis for the two reorientation method is also measured and noted in the last row of each table. The proposed method was implemented using Matlab 7.0 and the experiments on the performance between the two reorientation method is performed under an IBM compatible personal computer with $2.8 \mathrm{GHz}$ Intel Pentium D CPU and 1 GB RAM. 
Table 2: Experimental Results on Fig. 1 (a) and (d).

\begin{tabular}{|c|c|c|c|c|}
\hline & \multicolumn{2}{|c|}{ Applying TPS + RBF-NN } & \multicolumn{2}{|c|}{ Applying RT } \\
\hline & Left Ventricle & Right Ventricle & Left Ventricle & Right Ventricle \\
\hline $\begin{array}{l}\text { Adjusted } \\
\text { Orientation } \\
\text { Angle } \\
\end{array}$ & $\begin{array}{l}12.30 \text { degree } \\
\text { clockwise }\end{array}$ & $\begin{array}{l}11.78 \text { degree } \\
\text { anticlockwise }\end{array}$ & $\begin{array}{l}\text { 3.80 degree } \\
\text { clockwise }\end{array}$ & $\begin{array}{c}14.40 \text { degree } \\
\text { anticlockwise }\end{array}$ \\
\hline $\begin{array}{l}\text { Segmented } \\
\text { Ventricle }\end{array}$ & (a) & (b) & (c) & (d) \\
\hline Skeleton & (e) & (f) & (g) & (h) \\
\hline $\begin{array}{l}\text { TPS } \\
\text { Displaceme } \\
\text { nt values on } \\
\text { segmented } \\
\text { ventricle } \\
\text { boundary }\end{array}$ & & & & (l) \\
\hline $\begin{array}{l}\text { TPS } \\
\text { Displaceme } \\
\text { nt values on } \\
\text { Skeleton } \\
\text { boundary }\end{array}$ & (m) & & & (p) \\
\hline $\begin{array}{l}\text { Predicted } \\
\text { location of } \\
\text { tumor }\end{array}$ & (q) & & (r) & \\
\hline $\begin{array}{l}\text { Processing } \\
\text { time }\end{array}$ & & seconds & & ids \\
\hline
\end{tabular}


Table 3: Experimental Results on Fig. 1 (b) and (e).

\begin{tabular}{|c|c|c|c|c|}
\hline & \multicolumn{2}{|c|}{ Applying TPS + RBF-NN } & \multicolumn{2}{|c|}{ Applying RT } \\
\hline & Left Ventricle & Right Ventricle & Left Ventricle & Right Ventricle \\
\hline $\begin{array}{l}\text { Adjusted } \\
\text { Orientation } \\
\text { Angle } \\
\end{array}$ & $\begin{array}{l}\text { 5.80 degree } \\
\text { clockwise }\end{array}$ & $\begin{array}{l}8.44 \text { degree } \\
\text { clockwise }\end{array}$ & $\begin{array}{l}\text { 0.85 degree } \\
\text { clockwise }\end{array}$ & $\begin{array}{l}5.70 \text { degree } \\
\text { clockwise }\end{array}$ \\
\hline $\begin{array}{l}\text { Segmented } \\
\text { Ventricle }\end{array}$ & (a) & (b) & (c) & (d) \\
\hline Skeleton & (e) & (f) & (g) & (h) \\
\hline $\begin{array}{l}\text { TPS } \\
\text { Displacement } \\
\text { values on } \\
\text { segmented } \\
\text { ventricle } \\
\text { boundary }\end{array}$ & (i) & (j) & (k) & r \\
\hline $\begin{array}{l}\text { TPS } \\
\text { Displacement } \\
\text { values on } \\
\text { Skeleton } \\
\text { boundary }\end{array}$ & (m) & (n) & (0) & (p) \\
\hline $\begin{array}{l}\text { Predicted } \\
\text { location of } \\
\text { tumor }\end{array}$ & (q) & & (r) & \\
\hline $\begin{array}{l}\text { Processing } \\
\text { time }\end{array}$ & 838 & onds & & nds \\
\hline
\end{tabular}


Table 4: Experimental Results on Fig. 1 (c).

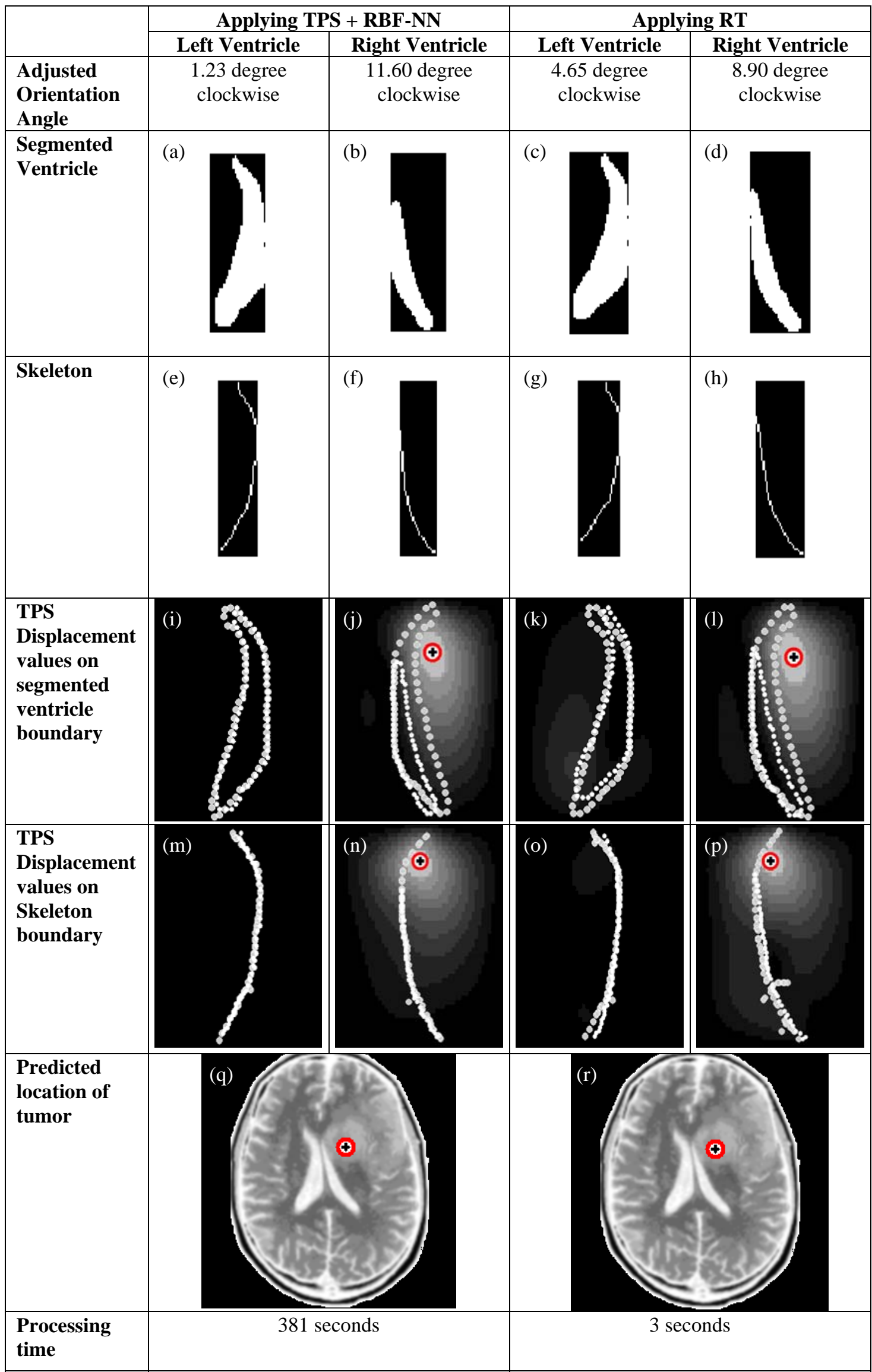


From the result of TPS analysis in Table 2 to 4, it can be seen that the orientated diagnostic lateral ventricle sampled boundary and its Skeleton using both the TPS and RBF-NN method and RT method is within reasonable degree from the template, although the degree of fit between the template and diagnostic sampled boundary points for the two methods is slightly different. Even with a difference of about 15 degree between the orientated right diagnostic in Table 1 using the two reorienting method, the location of the maximum TPS displacement does not differ significantly. This shows that the TPS analysis algorithm is able to give a stable result of the predicted location of the tumor even with a different reorientation algorithm which could have a slight difference in result between them.

Based on the processing time using the two reorientation method, it is estimated that the TPS and RBF-NN reorientation method is more than 100 times slower than the reorienting method using RT. This is mainly due to the processor intensive calculation required in the TPS analysis for each orientation of the diagnostic in the process of finding the orientation with the least TPS displacement. Therefore, based on our current experimental results, RT method of reorientation seems to be a better method as it is more efficient while giving a reasonable result.

\subsection{CONCLUSION}

Thin Plate Spline (TPS) is applied for three times in this study. It is at first served as a morphing transformation method to retrieve a interpolated image between standard template and the diagnostic images to reduce the effect of their boundary shape discrepancy.

As Radial Basis Neural Network (RBF-NN) is a type of feed forward neural network that can be used to approximate any function, by training it to function as a form of mean operator on an error measure, the orientation that gives the least output value from the network can be used as an indicator of the best orientation. From the results, it can be seen that the network is able to find the best orientation using Thin Plate Spline (TPS) displacement value as an error measure. However, it is found that using Radon Transform (RT) on Skeleton is much more efficient, while the similar results are maintained.

A 2-dimensional TPS can be used for coordinate transformation of a set of control points while affecting its surrounding data points with a smooth displacement change. In this paper, the original control points coordinate is the sampled template or normal brain ventricle reference boundary, and the final control points coordinate is the sampled Diagnostic or the abnormal brain ventricle boundary. By plotting the original data coordinate with an intensity value that represents the displacement value, it can be discerned that the area with the highest displacement value correlates with the approximate location of the tumor. The predicted result of the direction and location of the tumor is relevant to our subjective estimation. It is also found that using Thin Plate Spline (TPS) is effective in analyzing the lateral ventricle deformation caused by the compression from the brain tumor.

\section{REFERENCES}

[1] Graham, J., Babalola, K.O., Honer, W.G., Lang, D., Kopala, L., "Lateral asymmetry in the shape of brain ventricles in control and schizophrenia groups", Vandorpe, R., Biomedical Imaging: Macro to Nano, $3^{\text {rd }}$ IEEE International Symposium on Biomedical Imaging, Arlington VA., Vol. 6-9, April 2006, pp. 414 - 417.

[2] Marcel Prastawa, Elizabeth Bullitt, Sean Ho, Guido Gerig, “A brain tumor segmentation framework based on outlier detection”, Medical Image Analysis, Vol. 8, No. 3, September 2004, pg. 275-283.

[3] Image Gallery, Dreamstime, www.dreamstime.com , downloaded on 08 February, 2007.

[4] The Whole Brain Atlas, Harvard Medical School, http:/www.med.harvard.edu/AANLIB/home.html, downloaded on 14 September, 2006.

[5] Xiao K., Ho S.H., Salih Q., “A Study: Segmentation of Lateral Ventricles in Brain MRI using Fuzzy C Means Clustering with Gaussian Smoothing”, in Proceedings of the Joint Rough Set Symposium JRS2007 (Rough Sets, Fuzzy Sets, Data Mining \& Granular Computing RSFDGrC07), Toronto, May 14-16, 2007, pp. 161-170. 
[6] Xiao K, Ho S.H., Hassanien A.e., Nguyen V.D., Salih Q. , “Fuzzy C-Means Clustering with Adjustable Feature Weighting Distribution for Brain MRI Ventricles Segmentation”, in proceedings of the Ninth IASTED International Conference on Signal and Image Processing, Honolulu, Hawaii, USA, August 20-22, 2007, pp. 483-489.

[7] Christian Gaser, Igor Nenadic, Bradley R. Buchsbaum, Erin A. Hazlett, Monte S. Buchsbaum, "Deformation -Based Morphometry and Its Relation to Conventional Volumetry of Brain Lateral Ventricles in MRI”, NeuroImage, Vol. 13, No. 6, June 2001, pp. 1140-1145.

[8] Matthieu Ferrant, Arya Nabavi, Benoit Macq, P.M. Balck, ferenc A. Jolesz, Ron Kikinis, Simon K. Warfield, "Serial registration of intraoperative MR images of the brain”, Medical Image Analysis, Vol. 6, No. 4, 2002, December 2002, pp. 337-359.

[9] Torsten Rohlfing, Edith V. Sullivan, Adolf Pfefferbaum, "Deformation-based brain morphometry to track the course of alcoholism: Differences between intra subject and inter-subject analysis”, Neuroimaging, Vol. 146, No. 2, 31 March 2006, pp. 157-170.

[10] Kanti V. Mardia, Jose M. Angulo, Amaldo Goitia, "Synthesis of image deformation strategies”, Image and Vision Computing, Vol. 24, No. 1, 1 January 2006, pp. 1-12.

[11] Arun Ross, Sarat Dass, Anial Jain, “A deformable model for fingerprint Matching”, Pattern Recognition, Vol. 38, No. 1, January 2005, pp. 95-103.

[12] Hong-Dun Lin, Kang-Ping Lin, “3D deformable image processing and Integration”, International Congress Series, Vol. 1265, August 2004, pp. 39-48.

[13] Fred L. Bookstein, "Principal Warps: Thin-Plate Splines and the Decomposition of Deformation”, IEEE Transaction on Pattern Analysis and Machine Intelligence, Vol.11, No. 6, June 1989.

[14] Cecilia Di Ruberto, "Recognition of shapes by attributed skeletal graphs”, Pattern Recognition, Vol. 37, No.1, January 2004, pp. 21-31.

[15] J. Duchon, 1976, "Splines minimizing rotation invariant seminorms in sobolev Spaces”, In: Constructive Theory of Functions of Several Variables, Vol. 1, pp. 85-100.

[16] Faridoon Shabaninia,Mehdi Roopaei, Mehdi Fatemi, "Post-training on RBF neural networks", Nonlinear Analysis Hybrid Systems, Vol. 1, No. 4, December 2007, pp. 491-500.

[17] Afsar Saranli, Buyurman Baykal, "Complexity reduction in radial basis function (RBF) networks by using radial B-spline functions”, Neurocomputing, Vol. 18, No. 1-3, January 1998, pp. 183-194.

[18] Guido Bugmann, “Normalized Gaussian Radial Basis Function networks”, Neurocomputing, Vol. 20, No. 1-3, 31 August 1998, pp. 97-110.

[19] C. Harpham, C.W. Dawson, "The effect of different basis functions on a radial basis function network for time series prediction: A comparative study”, Neurocomputing, Vol. 69, No. 16-18, October 2006, pp. 2161 -2170 .

[20] Rajiv Kapoor, Deepak Bagai b, T.S. Kamal c, “A new algorithm for skew detection and correction”, Pattern Recognition Letters, Vol. 25, No. 11, 2004, pp. 1215 - 1229.

[21] Peiling Cui, Junhong Li, Quan Pan, Hongcai Zhang, "Rotation and scaling invariant texture classification based on Radon transform and multiscale analysis”, Pattern Recognition Letters, Vol. 27, No. 5, 1 April 2006, pp. 408-413. 


\section{BIOGRAPHY}

Kai Xiao, received his Master of Science in Information Technology from the University of Nottingham, Malaysia Campus in 2003. Currently, he is a postgraduate research student and $\mathrm{PhD}$ candidate in the Faculty of Engineering and Computer Science of the University of Nottingham, Malaysia Campus. His research interests include medical image processing, pattern recognition, and classification.

Sooi Hock Ho, received the Bachelor of Electrical Engineering from the University of Malaya in 1978, and the Master of Computer Science from University College, London in 1980. He worked in IBM Malaysia as a specialist in the areas of operating systems and networking from 1981 to 2001, and has since joined the University of Nottingham, Malaysia Campus. His research interests include digital image processing, image compression and pattern recognition.

Aboul Ella Hassanien, received his B.Sc. with honours in 1986 and M.Sc degree in 1993, both from Ain Shams University, Faculty of Science, Pure Mathematics and Computer Science Department, Cairo, Egypt. On September 1998, he received his doctoral degree from the Department of Computer Science, Graduate School of Science \& Engineering, Tokyo Institute of Technology, Japan. He is an associated Professor at Cairo University, Faculty of Computer and Information, IT Department. Currently, he is a visiting professor at Kuwait University, College of Business Administration, Quantitative and Information System Department. He has authored/coauthored over 80 research publications in peer-reviewed reputed journals and conference proceedings. He has served as the program committee member of various international conferences and reviewer for various international journals. Since 2004, he is actively involved as technical committee in the International Association of Science and Technology for Development (IASTED) for Image Processing and Signal Processing. He has received the excellence younger researcher award from Kuwait University for the academic year 2003/2004. He has guest edited many special issues for international scientific journals. He has directed many funded research projects. Dr. Abo was a member of the Interim Advisory Board committee of the International Rough Set Society. He is the editor and co-editor for more than six books in the area of rough computing, computational intelligence, and E-commerce. His research interests include, rough set theory, wavelet theory, X-ray Mammogram analysis, medical image analysis, fuzzy image processing and multimedia data mining. 